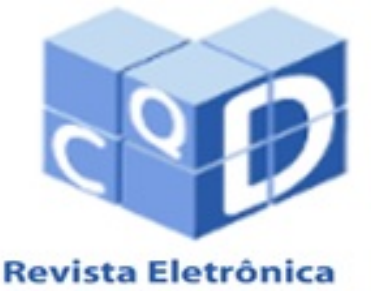

Revista Eletrônica

Paulista de Matemática

ISSN 2316-9664

Volume 9, jul. 2017

Fernando da Costa Gomes

Instituto Federal do MaranhãoCampus Pinheiro

fernando.costa@ifma.edu.br

\section{Uma demonstração da conjectura de Chen no espaço Euclidiano $E^{4}$}

A proof of Chen conjecture in the Euclidean space $E^{4}$.

\section{Resumo}

Seja $M^{2}$ uma superfície compacta bidimensional imersa no $m$ espaço Euclidiano $E^{m}$. A curvatura média total de $M^{2}$ é definida como sendo a integral $\int_{M^{2}} H^{2} d V$, onde $H$ e $d V$ denotam, respectivamente, a curvatura média e o elemento de volume da superfície $M^{2}$. Um problema interessante é encontrar o melhor limite inferior desta integral em termos dos invariantes geométricos ou topológicos de $M^{2}$. Muitos resultados tem sido obtidos acerca desse problema. Bang-Yen Chen (1981, p. 515) conjecturou que se $M^{2}$ é uma superfície bidimensional compacta de gênero $g \geq 1$ imersa no $m$-espaço Euclidiano $E^{m}$, então a integral do quadrado de sua curvatura média é pelo menos $2 \pi^{2}$. Neste trabalho, demonstraremos que essa conjectura é válida no caso do espaço Euclidiano 4-dimensional $E^{4}$.

Palavras-chave: Conjectura de Chen. Energia de Willmore. Números de Betti.

\begin{abstract}
Let $M^{2}$ be a two dimensional compact surface immersed in the Euclidean $m$-space $E^{m}$. The total mean curvature of $M^{2}$ is defined to be the integral $\int_{M^{2}} H^{2} d V$, where $H$ and $d V$ denote, respectively, the mean curvature and the volume element of the surface $M^{2}$. An interesting problem is to find the best possible lower bound of this integral in terms of the geometric or topologic invariants of $M^{2}$. There have been many results obtained on this problem. Bang-Yen Chen (1981, p. 515) conjectured that if $M^{2}$ is a two dimensional compact surface of genus $g \geq 1$ immersed in the Euclidean $m$-space $E^{m}$, so the integral of the square of the mean curvature is at least $2 \pi$. In this paper we prove this conjecture is true in the case of the Euclidean 4 -space $E^{4}$.

Keywords: Chen conjecture. Willmore's energy. Betti numbers.
\end{abstract}




\section{Introdução}

Na teoria clássica de superfícies fechadas (compactas e sem bordo) imersas em um espaço Euclidiano $m$-dimensional $E^{m}$, os dois invariantes geométricos básicos são a curvatura Gaussiana $K$ e a curvatura média $H$. A curvatura Gaussiana é um conceito intrínseco e sua integral nos fornece a conhecida fórmula de Gauss-Bonnet

$$
\int_{M^{2}} K d V=2 \pi \chi\left(M^{2}\right)
$$

onde $d V$ e $\chi\left(M^{2}\right)$ denotam, respectivamente, o elemento de volume e a característica de Euler da superfície $M^{2}$.

Segundo Willmore (1968), para uma 2-superfície $M^{2}$ imersa em $E^{m}$, a integral do quadrado da curvatura média, conhecida como a energia de Willmore, satisfaz

$$
W\left(M^{2}\right):=\int_{M^{2}} H^{2} \geq 4 \pi
$$

onde a igualdade ocorre se, e somente se, $M^{2}$ é uma 2-esfera em um 3-espaço afim. A energia de Willmore aparece naturalmente em alguns contextos físicos. Por exemplo, na Biomatemática ela aparece no modelo de Helfrich (1973) como um dos termos que contribuem para a energia das membranas celulares.

Chen (1979) mostrou que se $M^{2}$ é uma superfície compacta flat imersa em $E^{4}$, então

$$
W\left(M^{2}\right) \geq 2 \pi^{2}
$$

Uma extensão desse resultado para o caso $m$-dimensional $(m \geq 4)$ foi obtida pelo próprio Chen em 1981 e, além disso, foi proposta a seguinte conjectura.

Conjectura 1 Toda 2-superfície compacta $M^{2}$ de gênero $g \geq 1$ em $E^{m}$ satisfaz

$$
W\left(M^{2}\right) \geq 2 \pi^{2}
$$

O principal objetivo deste trabalho é demonstrar que a conjectura acima é válida para o caso em que $m=4$. Mais precisamente, demonstraremos o seguinte teorema.

Teorema 2 Seja $M^{2}$ uma 2-superfície compacta de gênero $g \geq 1$ imersa no espaço Euclidiano $E^{4}$. Então

$$
W\left(M^{2}\right) \geq 2 \pi^{2}
$$

\section{Método do referencial móvel}

Sejam $U$ um aberto do $\mathbb{R}^{n}$ e $\left(e_{1}, e_{2}, \ldots, e_{n}\right)$ campos diferenciáveis de vetores definidos em $U$ de tal modo que, para todo $q \in U$, se tenha $\left\langle e_{i}, e_{j}\right\rangle_{q}=\delta_{i j}$, onde $\delta_{i j}=0$ se $i \neq j$ e $\delta_{i j}=1$ se $i=j$, com $i, j=1, \ldots, n$. Um tal conjunto de campo de vetores é chamado um referencial ortonormal móvel em $U$ e será denotado por $\left\{e_{i}\right\}$. Doravante, omitiremos os adjetivos ortonormal e móvel, isto é, todos os referenciais serão ortonormais.

A partir do referencial $\left\{e_{i}\right\}$ podemos definir formas diferenciais lineares $\omega_{1}, \ldots, \omega_{n}$ pela condição $\omega_{i}\left(e_{j}\right)=\delta_{i j}$; em outras palavras, em cada ponto $q \in U$, a base $\left\{\left(\omega_{i}\right)_{q}\right\}$ é a base 
dual de $\left\{\left(e_{i}\right)_{q}\right\}$. O conjunto das formas diferenciais $\left\{\omega_{i}\right\}$ é chamado o correferencial associado ao referencial $\left\{e_{i}\right\}$.

Cada campo $e_{i}$ é uma aplicação diferenciável $e_{i}: U \subset \mathbb{R}^{n} \rightarrow \mathbb{R}^{n}$. A diferencial $\left(d e_{i}\right)_{q}$ : $\mathbb{R}^{n} \rightarrow \mathbb{R}^{n}$, em $q \in U$, é uma aplicação linear. Portanto, para todo $v \in \mathbb{R}^{n}$, podemos escrever

$$
\left(d e_{i}\right)_{q}(v)=\sum_{j}\left(\omega_{i j}\right)_{q}(v) e_{j} .
$$

As expressões $\left(\omega_{i j}\right)_{q}(v)$, acima definidas, dependem linearmente de $v$ e diferenciavelmente de $q$. Portanto, $\left(\omega_{i j}\right)_{q}$ é uma forma linear em $\mathbb{R}^{n}$. Como $e_{i}$ é um campo diferenciável, então $\omega_{i j}$ é uma forma diferenciável linear. Com estes significados em mente, escreveremos

$$
d e_{i}=\sum_{j} \omega_{i j} e_{j}
$$

como definição das formas $\omega_{i j}$, que são chamadas formas de conexão do $\mathbb{R}^{n}$ no referencial $\left\{e_{i}\right\}$.

Diferenciando a expressão $\left\langle e_{i}, e_{j}\right\rangle_{q}=\delta_{i j}$, obtemos

$$
0=\left\langle d e_{i}, e_{j}\right\rangle_{q}+\left\langle e_{i}, d e_{j}\right\rangle_{q}=\omega_{i j}+\omega_{j i},
$$

isto é, as formas de conexão $\omega_{i j}$ são antissimétricas nos índices $i, j$.

Teorema 3 (Equações de Estrutura do $\mathbb{R}^{n}$ ) Seja $\left\{e_{i}\right\}$ um referencial em um aberto $U \subset \mathbb{R}^{n}$. Sejam $\left\{\omega_{i}\right\}$ o correferencial associado a $\left\{e_{i}\right\}$ e $\omega_{i j}$ as formas de conexão de $U$ no referencial $e_{i}$. Então,

$$
\begin{gathered}
d \omega_{i}=\sum_{k} \omega_{k} \wedge \omega_{k i}, \\
d \omega_{i j}=\sum_{k} \omega_{i k} \wedge \omega_{k j}, \quad k=1, \ldots, n .
\end{gathered}
$$

Demonstração: Sejam $a_{1}=(1,0, \ldots, 0), a_{2}=(0,1,0, \ldots, 0), \ldots, a_{n}=(0,0, \ldots, 0,1)$ a base canônica do $\mathbb{R}^{n}$ e $x_{i}: U \rightarrow \mathbb{R}$ a função que faz corresponder a cada ponto $q=\left(x_{1}, \ldots, x_{n}\right) \in U$ a sua $i$-ésima coordenada. Então, $d x_{i}$ é uma forma diferencial em $U$ e, como $d x_{i}\left(a_{j}\right)=\delta_{i j}$, concluímos que $\left\{d x_{i}\right\}$ é o correferencial associado ao referencial $\left\{a_{i}\right\}$. O referencial dado se exprime em termos dos $a_{i}$ por

$$
e_{i}=\sum_{j} \beta_{i j} a_{j}
$$

onde os $\beta_{i j}$ são funções diferenciáveis em $U$ e, para cada $q \in U$, a matriz $\left(\beta_{i j}(q)\right)$ é uma matriz ortogonal. Como $\omega_{i}\left(e_{j}\right)=\delta_{i j}$, temos

$$
\omega_{i}=\sum_{j} \beta_{i j} d x_{j}
$$

Diferenciando (4), obtemos

$$
d e_{i}=\sum_{k} d \beta_{i k} a_{k}=\sum_{k} d \beta_{i j} \sum_{j} \beta_{j k} e_{j} .
$$

Como $d e_{i}=\sum_{j} \omega_{i j} e_{j}$, concluímos que

$$
\omega_{i j}=\sum_{k} d \beta_{i k} \beta_{j k}
$$


ou seja,

$$
\sum_{j} \omega_{i j} \beta_{j s}=\sum_{j k} d \beta_{i k} \beta_{j k} \beta_{j s}=d \beta_{i s}, \quad s=1, \ldots, n .
$$

Por fim, diferenciando exteriormente (5) e usando (7), obtemos

$$
d \omega_{i}=\sum_{j} d \beta_{i j} \wedge d x_{j}=\sum_{j, k} \omega_{i k} \beta_{k j} \wedge d x_{j}=\sum_{k} \omega_{k} \wedge \omega_{k i},
$$

que é a primeira equação de estrutura (2).

Diferenciando (6) e usando (7), obtemos

$$
\begin{aligned}
d \omega_{i j} & =-\sum_{k} d \beta_{i k} \wedge d \beta_{j k} \\
& =-\sum_{k}\left\{\left(\sum_{l=1}^{n} \omega_{i l} \beta_{l k}\right) \wedge\left(\sum_{s} \omega_{j s} \beta_{s k}\right)\right\} \\
& =-\sum_{s} \omega_{i s} \wedge \omega_{j s} \\
& =\sum_{k} \omega_{i k} \wedge \omega_{k j}
\end{aligned}
$$

que é a segunda equação de estrutura (3).

De modo inteiramente análogo ao que foi feito em $\mathbb{R}^{n}$, podemos definir, mais geralmente, um referencial ortonormal móvel em um aberto $U$ de uma variedade Riemanniana $M^{n}$ qualquer. Para finalizarmos esta seção, apresentaremos o importante lema de Cartan e demonstraremos a existência e unicidade das formas de conexão.

Lema 4 (Cartan) Sejam $V$ um espaço vetorial de dimensão ne $\omega_{1}, \ldots, \omega_{r}: V \rightarrow \mathbb{R}, r \leq$ $n$, formas lineares de $V$ linearmente independentes. Suponhamos que existam formas lineares $\theta_{1}, \ldots, \theta_{r}: V \rightarrow \mathbb{R}$ satisfazendo a seguinte condição,

$$
\sum_{i=1}^{r} \omega_{i} \wedge \theta_{i}=0
$$

Então,

$$
\theta_{i}=\sum_{j} a_{i j} \omega_{j}, \quad i, j=1, \ldots, r, \quad a_{i j}=a_{j i}
$$

Demonstração: Completemos as formas $\omega_{1}, \ldots, \omega_{r}$ em uma base $\omega_{1}, \ldots, \omega_{r}, \omega_{r+1}, \ldots, \omega_{n}$ de $V^{*}$ (espaço dual de $V$ ) e escrevamos

$$
\theta_{i}=\sum_{j=1}^{r} a_{i j} \omega_{j}+\sum_{l=r+1}^{n} b_{i l} \omega_{l} .
$$

Basta agora observarmos que a condição $\sum_{i} \omega_{i} \wedge \theta_{i}=0$ implica em

$$
\begin{aligned}
0 & =\sum_{i=1}^{r} \omega_{i} \wedge \theta_{i} \\
& =\sum_{i=1}^{r} \omega_{i} \wedge \sum_{j=1}^{r} a_{i j} \omega_{j}+\sum_{i=1}^{r} \omega_{i} \wedge \sum_{l=r+1}^{n} b_{i l} \omega_{l} \\
& =\sum_{i<j}\left(a_{i j}-a_{j i}\right) \omega_{i} \wedge \omega_{j}+\sum_{i<l} b_{i l} \omega_{i} \wedge \omega_{l} .
\end{aligned}
$$


Como os $\omega_{k} \wedge \omega_{s}, \quad k<s, \quad k, s=1, \ldots, n$, são linearmente independentes, concluímos que $a_{i j}=a_{j i}$ e $b_{i l}=0$.

Lema 5 Sejam $M^{n}$ uma variedade Riemanniana, $q \in M^{n}$ e $U \subset M^{n}$ uma vizinhança de $q$. Sejam $\left(e_{1}, \ldots, e_{n}\right)$ um referencial móvel em $U$ e $\omega_{1}, \ldots, \omega_{n}$ o correferencial associado a $\left\{e_{i}\right\}$. Suponha que exista em $U$ um conjunto de 1-formas diferenciais $\omega_{i j}$ satisfazendo as condições

$$
\omega_{i j}=-\omega_{j i} \text { e d } \omega_{j}=\sum_{k} \omega_{k} \wedge \omega_{k j}
$$

Então um tal conjunto é único.

Demonstração: Suponhamos que exista outro conjunto de formas $\bar{\omega}_{i j}$ com

$$
\bar{\omega}_{i j}=-\bar{\omega}_{j i}, \quad d \omega_{j}=\sum_{k} \omega_{k} \wedge \bar{\omega}_{k j}
$$

Então, $\sum_{k} \omega_{k} \wedge\left(\bar{\omega}_{k j}-\omega_{k j}\right)=0$ e, pelo lema de Cartan,

$$
\bar{\omega}_{k j}-\omega_{k j}=\sum_{i} B_{k i}^{j} \omega_{i}, \quad B_{k i}^{j}=B_{i k}^{j}
$$

Observemos que,

$$
\bar{\omega}_{k j}-\omega_{k j}=\sum_{i} B_{k i}^{j} \omega_{i}=-\left(\bar{\omega}_{j k}-\omega_{j k}\right)=-\sum_{i} B_{j i}^{k} \omega_{i}
$$

e, como os $\omega_{i}$ são linearmente independentes, então $B_{k i}^{j}=-B_{j i}^{k}$. Usando as simetrias obtidas, concluímos que

$$
B_{j i}^{k}=-B_{k i}^{j}=-B_{i k}^{j}=B_{j k}^{i}=B_{k j}^{i}=-B_{i j}^{k}=-B_{j i}^{k}=0
$$

ou seja, $\bar{\omega}_{k j}=\omega_{k j}$.

Lema 6 Escolhido um referencial $\left\{e_{i}\right\}$ em um aberto $U \subset M^{n}$ de uma variedade Riemanniana $M^{n}$, existe em $U$ um conjunto de formas diferenciais $\omega_{i j}$ que satisfazem

$$
\omega_{i j}=-\omega_{j i} \text { e d } \omega_{j}=\sum_{k} \omega_{k} \wedge \omega_{k j}
$$

Demonstração: Dado um ponto $q \in M^{n}$, o conjunto $\left\{\omega_{i} \wedge \omega_{j} ; i<j, i, j=1, \ldots, n\right\}$ forma uma base para o espaço $\Lambda^{2}\left(T_{q} M^{n}\right)^{*}$ das formas bilineares alternadas de $T_{q} M^{n} \times T_{q} M^{n}$, onde $T_{q} M^{n}$ denota o espaço tangente a $M^{n}$ em $q$. Assim, podemos escrever

$$
d \omega_{j}=\sum_{k<i} A_{k i}^{j} \omega_{k} \wedge \omega_{i} ; \text { com } A_{k i}^{j}=-A_{i k}^{j}
$$

Queremos determinar funções $C_{k j}^{i}=-C_{j k}^{i}$ tais que as formas diferenciais

$$
\omega_{k j}=\sum_{i} C_{k j}^{i} \omega_{i}
$$


satisfaçam (10). Se tais formas existirem, então de (10) e (11) teremos

$$
\begin{aligned}
d \omega_{j} & =\sum_{k<i} A_{k i}^{j} \omega_{k} \wedge \omega_{i}=\sum_{k} \omega_{k} \wedge\left(\sum_{i} C_{k j}^{i} \omega_{i}\right) \\
& =\sum_{k<i}\left(C_{k j}^{i}-C_{i j}^{k}\right) \omega_{k} \wedge \omega_{i} .
\end{aligned}
$$

Igualando os coeficientes de termos correspondentes nas equações acima, obtemos

$$
\begin{aligned}
A_{k i}^{j} & =C_{k j}^{i}-C_{i j}^{k} \\
A_{i j}^{k} & =C_{i k}^{j}-C_{j k}^{i} \\
A_{k j}^{i} & =C_{k i}^{j}-C_{j i}^{k} .
\end{aligned}
$$

Adicionando membro a membro as igualdades acima, encontraremos a seguinte condição necessária para a existência dos $C_{k j}^{i}$,

$$
C_{k j}^{i}=\frac{1}{2}\left(A_{k i}^{j}+A_{i j}^{k}+A_{k j}^{i}\right)
$$

Então, basta definirmos $C_{k j}^{i}$ como em (13) e as formas $\omega_{i j}$ por (12).

\section{Subvariedades em um espaço Euclidiano}

Seja $f: M^{n} \rightarrow E^{n+p}$ uma imersão de uma variedade suave compacta sem bordo $n$-dimensional $M^{n}$ em um espaço Euclidiano $E^{n+p}$ de dimensão $n+p$.

Ao longo desta seção, identificaremos $M^{n}$ com sua imagem imersa e convencionaremos os seguintes domínios de índices: $1 \leq i, j, k \leq n ; 1 \leq \alpha, \beta, \gamma \leq p ; n+1 \leq r, s, t \leq n+p ; 1 \leq$ $A, B, C \leq n+p$. Além disso, denotaremos por $N_{q} M^{n}$ o espaço normal a $M^{n}$ em $q$.

Consideremos no fibrado tangente $T\left(E^{n+p}\right)$ um referencial ortonormal local $\left(e_{1}, \ldots, e_{n+p}\right)$ com a propriedade que, quando restritos a um aberto $U$ de $M^{n}$, os vetores $\left(e_{1}, \ldots, e_{n}\right)$ sejam tangentes a $U$ e os vetores $\left(e_{n+1}, \ldots, e_{n+p}\right)$ sejam normais a $U$. Denotemos por $\left(\omega_{1}, \ldots, \omega_{n+p}\right)$ seu respectivo correferencial. Como vimos na Seção 1, existe uma única 1-forma de conexão, $\left(\omega_{A B}\right)$, tal que

$$
d \omega_{A}=\sum_{B} \omega_{A B} \wedge \omega_{B}, \quad \omega_{A B}+\omega_{B A}=0, \quad d \omega_{A B}=\sum_{C} \omega_{A C} \wedge \omega_{C B} .
$$

Restringindo estas formas a $M^{n}$, temos $\omega_{r}=0$, para todo $r$. Assim,

$$
0=d \omega_{r}=\sum_{i} \omega_{r i} \wedge \omega_{i}, \forall r
$$

Pelo lema de Cartan, temos

$$
\omega_{r i}=\sum_{j} h_{i j}^{r} \omega_{j}, \quad h_{i j}^{r}=h_{j i}^{r}, \forall i, j, r .
$$


A primeira e a segunda forma fundamental são, respectivamente, dadas por

$$
I=\sum_{i}\left(\omega_{i}\right)^{2} \quad \text { e } \quad I I=\sum_{i, j, r} h_{i j}^{r} \omega_{i} \omega_{j} e_{r}
$$

O operador de forma $A_{e}$ de $M^{n}$ com relação ao vetor normal $e \in N_{q} M^{n}$ é o operador autoadjunto em $T_{q} M^{n}$ correspondente à forma quadrática $I I_{e}=\langle I I, e\rangle$. A matriz de $A_{e_{r}}$ com relação à base adaptada $\left\{e_{1}, \ldots, e_{n+p}\right\}$ é $L_{r}=\left(h_{i j}^{r}\right)_{n \times n}$.

Além disso, podemos representar o campo vetor curvatura média $\xi$, a curvatura média $H$ e o comprimento ao quadrado da segunda forma fundamental $S$ da seguinte maneira

$$
\xi=\sum_{r} H_{r} e_{r}, \quad H=|\xi|, \quad S=\sum_{i, j, r}\left(h_{i j}^{r}\right)^{2}
$$

onde $H_{r}=\frac{1}{n} \sum_{i} h_{i i}^{r}$ para todo $r$.

Denotemos por $B_{\nu}$ o fibrado normal unitário de $f\left(M^{n}\right)$ em $E^{n+p}$, isto é,

$$
B_{\nu}=\left\{(x, \nu(x)) \mid x \in M^{n} \text { e } \nu(x) \in N_{f(x)} M^{n}, \operatorname{com}\langle\nu(x), \nu(x)\rangle=1\right\} .
$$

Notemos que $B_{\nu}$ é localmente um fibrado de esferas $(p-1)$-dimensionais sobre $f\left(M^{n}\right)$ e é localmente uma variedade diferenciável de dimensão $n+p-1$.

Existe uma forma diferenciável $d \sigma_{p-1}$ de grau $p-1$ em $B_{\nu}$ tal que, quando restrita a uma fibra, é o elemento de volume da esfera de vetores normais e unitários em um ponto $x \in M^{n}$, denotada por $S_{x}^{p-1}$.

Com efeito, podemos pensar em $f=e_{n+p}$ como vetor posição da esfera $S_{x}^{p-1}$. Assim, das equações (1) e (2) obtemos

$$
d f=\sum_{\alpha=1}^{p-1} \omega_{n+\alpha} e_{n+\alpha} \quad \text { e } \quad d e_{n+p}=\sum_{\alpha=1}^{p-1} \omega_{n+p, n+\alpha} e_{n+\alpha}
$$

donde segue que,

$$
\omega_{n+p, n+\alpha}=\omega_{n+\alpha} .
$$

Desse modo, o elemento de volume de $S_{x}^{p-1}$ no referencial acima é dado pela $(p-1)$-forma

$$
d \sigma_{p-1}=\omega_{n+1} \wedge \cdots \wedge \omega_{n+p-1}=\omega_{n+p, n+1} \wedge \cdots \wedge \omega_{n+p, n+p-1} .
$$

Por outro lado, o elemento de volume de $M^{n}$ pode ser representado pela $n$-forma $d V=\omega_{1} \wedge \cdots \wedge$ $\omega_{n}$. Como $B_{\nu}$ é uma variedade diferenciável $(n+p-1)$-dimensional, então a $(n+p-1)$-forma dada por

$$
d V \wedge d \sigma_{p-1}=\omega_{1} \wedge \cdots \wedge \omega_{n} \wedge \omega_{n+1} \wedge \cdots \wedge \omega_{n+p-1}
$$

pode ser considerada como o elemento de volume de $B_{\nu}$.

Em um ponto arbitrário $(x, e) \in B_{\nu}$, denotemos $A_{e}=\left(A_{i j}\right)$. Definimos a k-ésima curvatura média $K_{k}(x, e)$ em $(x, e)$ por

$$
\operatorname{det}\left(\delta_{i j}+t A_{i j}\right)=1+\sum_{k}\left(\begin{array}{l}
n \\
k
\end{array}\right) K_{k}(x, e) t^{k},
$$

onde $\delta_{i j}$ é o delta de Kronecker, $t$ é um parâmetro e

$$
\left(\begin{array}{l}
n \\
k
\end{array}\right)=\frac{n !}{k !(n-k) !}
$$


Quando $k=n$, a $k$-ésima curvatura média coincide com a curvatura de Lipschitz-Killing em $(x, e)$. Chamamos a integral

$$
K_{k}^{*}(x):=\int_{S_{x}^{p-1}}\left|K_{k}(x, e)\right|^{n / k} d \sigma_{p-1}(e),
$$

a $k$-ésima curvatura absoluta total de $M^{n}$ em $x$. A $k$-ésima curvatura absoluta total com relação a $f$ é definida por

$$
T A_{k}(f):=\frac{1}{c_{n+p-1}} \int_{M^{n}} K_{k}^{*} d V
$$

onde $c_{n+p-1}$ denota o volume da esfera unitária $(n+p-1)$-dimensional.

O lema, a seguir, estabelece um limite inferior para $T A_{n}(f)$ em termos dos $i$-ésimos números de Betti $\beta_{i}\left(M^{n}\right)$ de $M^{n}$ (confira a Seção 4 para a definição de $\beta_{i}\left(M^{n}\right)$ ).

Lema 7 Seja $f: M^{n} \rightarrow E^{n+p}$ uma imersão de uma variedade fechada em $E^{n+p}$. Então,

$$
T A_{n}(f) \geq \sum_{i=0}^{n} \beta_{i}\left(M^{n}\right)
$$

onde $\beta_{i}\left(M^{n}\right)$ é o i-ésimo número de Betti.

Demonstração: Vide (CHERN; LASHOF, 1958, p. 5).

\section{Complexos simpliciais}

Dizemos que $a_{0}, a_{1}, \ldots, a_{r}$ em $\mathbb{R}^{n}$ são pontos independentes quando os vetores

$$
a_{1}-a_{0}, a_{2}-a_{0}, \ldots, a_{r}-a_{0}
$$

são linearmente independentes. Esta definição não depende da ordem em que os pontos foram listados inicialmente, como se vê sem dificuldade.

Exemplo 8 Dois pontos distintos são independentes. Três pontos são independentes quando são não-colineares e quatro pontos independentes são pontos não-coplanares. Se $\left\{e_{1}, \ldots, e_{n}\right\}$ é a base canônica do $\mathbb{R}^{n}$, então os pontos $0, e_{1}, \ldots, e_{n}$ são independentes. O número máximo de pontos independentes em $\mathbb{R}^{n}$ é $n+1$.

Uma combinação afim de pontos $a_{0}, a_{1}, \ldots, a_{r}$ em $\mathbb{R}^{n}$ é uma expressão do tipo

$$
p=\alpha_{0} \cdot a_{0}+\alpha_{1} \cdot a_{1}+\cdots+\alpha_{r} \cdot a_{r},
$$

com $\alpha_{0}+\alpha_{1}+\cdots+\alpha_{r}=1$. Se, além disto, tivermos $\alpha_{0} \geq 0, \alpha_{1} \geq 0, \ldots, \alpha_{r} \geq 0$, diremos que $p$ é uma combinação convexa dos pontos $a_{0}, a_{1}, \ldots, a_{r}$.

Um conjunto $X \subset \mathbb{R}^{n}$ é convexo se, e somente se, toda combinação convexa de elementos de $X$ ainda pertence a $X$.

O conjunto de todas as combinações convexas de um conjunto arbitrário $X \subset \mathbb{R}^{n}$ é um conjunto convexo. Ele é chamado a envoltória convexa de $X$ e está contido em qualquer conjunto convexo que contenha $X$. Neste sentido, a envoltória convexa de $X$ é o menor conjunto convexo 
contendo $X$. Podemos descrevê-la como a interseção de todos os conjuntos convexos que contém $X$.

Sejam $a_{0}, a_{1}, \ldots, a_{k}$ pontos independentes em $\mathbb{R}^{n}$. O simplexo $k$-dimensional (ou $k$-simplexo) que tem estes pontos como vértices é o conjunto $\sigma=\left\langle a_{0}, a_{1}, \ldots, a_{k}\right\rangle$ de todas as combinações convexas $p=\sum_{i=0}^{k} \alpha_{i} a_{i}$, ou seja, é a envoltória convexa do conjunto $\left\{a_{0}, a_{1}, \ldots, a_{k}\right\}$. O número $k$ é chamado a dimensão do simplexo.

Fixado um subconjunto $\left\{i_{0}, i_{1}, \ldots, i_{j}\right\} \subset\{0,1, \ldots, k\}$, o simplexo $\left\langle a_{i_{0}}, a_{i_{1}}, \ldots, a_{i_{j}}\right\rangle$ é chamado uma face de $\sigma$. Em particular, cada vértice de $\sigma$ é uma face de dimensão zero. Para cada $i=0, \ldots, k$, a face $\sigma_{(i)}=\left\langle a_{0}, a_{1}, \ldots, \widehat{a}_{i}, \ldots, a_{k}\right\rangle$ chama-se a face oposta ao vértice $a_{i}$. Se $\tau$ é uma face de $\sigma$, escreveremos $\tau \preceq \sigma$.

Um poliedro é um subconjunto $K \subset \mathbb{R}^{n}$, no qual foi especificada uma coleção finita de simplexos de $\mathbb{R}^{n}$, chamados os simplexos de $K$, de modo que as condições abaixo são satisfeitas:

1. Todo ponto de $K$ pertence a algum simplexo de $K$ (ou seja, $K$ é a reunião dos seus simplexos);

2. Toda face de um simplexo de $K$ é ainda um simplexo de $K$;

3. Se $\sigma$ e $\rho$ são simplexos de $K$, então $\sigma \cap \rho$ é vazio ou é uma face comum a $\sigma$ e $\rho$ (e portanto é um simplexo de $K$ ).

Exemplo 9 O poliedro mais simples é um simplexo, juntamente com suas faces. Em dimensões zero, um, dois e três são, respectivamente, um ponto, um segmento de reta, um triângulo e um tetraedro.

Consideremos um $k$-simplexo $\sigma$, o qual é a envoltória de um conjunto $A$ de $k+1$ pontos independentes $a_{0}, \ldots, a_{k}(d \geq k)$ em algum espaço Euclidiano $\mathbb{R}^{d}$. Neste caso, dizemos que $A$ gera o simplexo $\sigma$.

Uma orientação de $\sigma$ é induzida por uma ordenação de seus vértices, denotada por $\left\langle a_{0} \cdots a_{k}\right\rangle$, como segue: Para qualquer permutação $\pi$ de $0, \ldots, k$, temos

$$
\left\langle a_{\pi(0)} \cdots a_{\pi(k)}\right\rangle=(-1)^{\operatorname{sign}(\pi)}\left\langle a_{0}, \cdots, a_{k}\right\rangle,
$$

onde o $\operatorname{sign}(\pi)$ é o número de transposições de $\pi$ (logo, cada simplexo tem duas orientações distintas). Um simplexo junto com uma escolha específica de orientação é chamado simplexo orientado.

Um complexo simplicial $K$ é um conjunto finito de simplexos em algum espaço Euclidiano $\mathbb{R}^{n}$, tal que (i) se $\sigma$ é um simplexo de $K$ e $\tau$ é uma face de $\sigma$, então $\tau$ é um simplexo de $K$, e (ii) se $\sigma$ e $\tau$ são simplexos de $K$, então $\sigma \cap \tau$ é ou vazia ou uma face comum de $\sigma$ e $\tau$. A dimensão de $K$ é o máximo das dimensões de seus simplexos. Se $d$ é a dimensão de $K$, diremos que $K$ é um $d$-complexo simplicial. A união de todos os simplexos de $K$ induzidos com a topologia subespaço de $\mathbb{R}^{m}$ será denotada por $|K|$.

O $i$-esqueleto de $K$, denotado por $K^{i}$, é a união de todos os simplexos de $K$ de dimensão no máximo $i$. Um subcomplexo $L$ de $K$ é um subconjunto de $K$ que é um complexo simplicial. Uma triangulação de um espaço topológico $X$ é um par $(K, h)$, onde $K$ é um complexo simplicial e $h$ é um homeomorfismo de $|K|$ em X. 
A característica de Euler de um $d$-complexo simplicial $K$, denotada por $\chi(K)$, é o número

$$
\sum_{i=0}^{d}(-1)^{i} \alpha_{i}
$$

onde $\alpha_{i}$ é o número de $i$-simplexos de $K$.

\section{Espaços de cadeia e homologia simplicial}

Seja $K$ um complexo simplicial. Uma $k$-cadeia simplicial é uma soma formal do tipo $\sum_{j} a_{j} \sigma_{j}$ sobre os $k$-simplexos orientados $\sigma_{j}$ em $K$, com coeficientes $a_{j}$ no corpo $\mathbb{Q}$ dos números racionais. Além disso, por definição, $-\sigma=(-1) \sigma$ é o simplexo obtido de $\sigma$ invertendo-se sua orientação.

Com as definições canônicas de adição e multiplicação por escalar, o conjunto de todas as cadeias $k$-simpliciais forma um espaço vetorial $C_{k}(K, \mathbb{Q})$, chamado espaço vetorial de $k$-cadeias simpliciais de $K$, que é o espaço vetorial livre gerado pelos $k$-simplexos. A dimensão desse espaço vetorial é igual ao número de $k$-simplexos de $K$. Portanto, a característica de Euler de um complexo simplicial $d$-dimensional $K$ pode ser expressada como uma soma alternada das dimensões dos espaços de $k$-cadeias,

$$
\chi(K)=\sum_{i=0}^{d}(-1)^{i} \operatorname{dim} C_{k}(K, \mathbb{Q}) .
$$

Seja $\left\langle v_{i_{0}} \cdots v_{i_{h}} \cdots v_{i_{k}}\right\rangle$ um $k$-simplexo. Usaremos a notação $\left\langle v_{i_{0}} \cdots \hat{v}_{i_{h}} \cdots v_{i_{k}}\right\rangle$ para indicar a omissão do termo $v_{i_{h}}$.

O operador de bordo $\partial_{k}: C_{k}(K, \mathbb{Q}) \rightarrow C_{k-1}(K, \mathbb{Q})$ é definido como segue. Dado um único $k$-simplexo $\sigma=\left\langle v_{i_{0}} \cdots v_{i_{k}}\right\rangle, k>0$, pomos

$$
\partial_{k} \sigma=\sum_{h=0}^{k}(-1)^{h}\left\langle v_{i_{0}} \cdots \hat{v}_{i_{h}} \cdots v_{i_{k}}\right\rangle,
$$

e então estendemos linearmente $\partial_{k}$ pondo

$$
\partial_{k}\left(\sum_{j} a_{j} \sigma_{j}\right)=\sum_{j} a_{j} \partial_{k} \sigma_{j} .
$$

Por consistência definimos $C_{-1}(K, \mathbb{Q})=0$ e $\partial_{0}: C_{0}(K, \mathbb{Q}) \rightarrow C_{-1}(K, \mathbb{Q})$ como sendo a aplicação nula. O operador de bordo é uma aplicação linear entre espaços vetoriais e satisfaz a relação $\partial_{k} \partial_{k+1}=0$.

$\mathrm{O}$ espaço vetorial $Z_{k}(K, \mathbb{Q})=\operatorname{ker} \partial_{k}$ é chamado espaço vetorial de $k$-ciclos simpliciais. O espaço vetorial $B_{k}(K, \mathbb{Q})=\mathrm{im} \partial_{k+1}$ é chamado espaço vetorial de $k$-bordos simpliciais. Como o bordo de um bordo é $0, B_{k}(K, \mathbb{Q})$ é um subespaço de $Z_{k}(K, \mathbb{Q})$.

$\mathrm{O}$ espaço vetorial quociente

$$
H_{k}(K, \mathbb{Q})=Z_{k}(K, \mathbb{Q}) / B_{k}(K, \mathbb{Q})
$$

é o k-ésimo espaço vetorial de homologia de $K$. Dois $k$-ciclos $\alpha$ e $\beta$ são $k$-homólogos se a diferença entre eles é um $k$-bordo, isto é, se existe uma $(k+1)$-cadeia $\gamma$ tal que $\alpha-\beta=\partial_{k+1} \gamma$. A classe de homologia de $\alpha \in Z_{k}(K, \mathbb{Q})$ é denotada por $[\alpha]$. 
Os coeficientes de cadeias simpliciais que consideramos até agora foram os números racionais. Normalmente, esses coeficientes são tomados em um anel, como o conjunto dos inteiros. Neste caso, obtém-se grupos de homologia, em vez de espaços vetoriais de homologia. Assim, $H_{k}(K, \mathbb{Z})$ é chamado de $k$-ésimo grupo de homologia do complexo $K$.

O k-ésimo número de Betti de um complexo simplicial $K$, denotado por $\beta_{k}(K, \mathbb{Q})$, é a dimensão de $H_{k}(K, \mathbb{Q})$. Em particular,

$$
\beta_{k}(K, \mathbb{Q})=\operatorname{dim} Z_{k}(K, \mathbb{Q})-\operatorname{dim} B_{k}(K, \mathbb{Q}) .
$$

\section{Demonstração do resultado principal}

Demonstração: (Do Teorema 2). O gênero $g$ e a característica de Euler $\chi\left(M^{2}\right)$ de uma 2superfície estão relacionados pela equação $\chi\left(M^{2}\right)=2-2 g$. Como, por hipótese, $g \geq 1$, então $\chi\left(M^{2}\right) \leq 0$. Da fórmula de Gauss-Bonnet, segue que $M^{2}$ tem curvatura Gaussiana $K \leq 0$. Levando-se em conta que "a curvatura escalar normalizada de uma 2-superfície coincide com sua curvatura Gaussiana"(HOU, 1998, p. 503) e usando uma desigualdade devida a Chen (1973, p. 641), obtemos

$$
\int_{M^{2}} H^{2} d V \geq \frac{\pi^{2}}{2} \cdot\left(\frac{1}{c_{3}} \int_{M^{2}} K_{2}^{*} d V\right)+\frac{\pi}{4} \int_{M^{2}} K d V
$$

onde $c_{3}$ denota o volume da esfera unitária 3-dimensional de $E^{4}$.

Da identidade $\chi\left(M^{2}\right)=\beta_{0}\left(M^{2}\right)-\beta_{1}\left(M^{2}\right)+\beta_{2}\left(M^{2}\right)$, do Lema 7 e de $(16)$, temos

$$
\begin{aligned}
\int_{M^{2}} H^{2} d V & \geq \frac{\pi^{2}}{2} \cdot\left[\beta_{0}\left(M^{2}\right)+\beta_{1}\left(M^{2}\right)+\beta_{2}\left(M^{2}\right)\right]+\frac{\pi^{2}}{2} \cdot\left[\beta_{0}\left(M^{2}\right)-\beta_{1}\left(M^{2}\right)+\beta_{2}\left(M^{2}\right)\right] \\
& =\pi^{2}\left[\beta_{0}\left(M^{2}\right)+\beta_{2}\left(M^{2}\right)\right] \\
& =2 \pi^{2}
\end{aligned}
$$

onde usamos na última igualdade o fato de que $\beta_{0}\left(M^{2}\right)=\beta_{2}\left(M^{2}\right)=1$ para toda superfície bidimensional compacta, conforme Otsuki (1966).

\section{Referências}

CHEN, B. Y. On the total curvature of immersed manifolds, III: surfaces in Euclidean 4-space, Amer. J. Math, v. 95, n. 3, p. 636-642, 1973.

CHEN, B. Y. On the total curvature of immersed manifolds, IV: spectrum and total mean curvature. Bull. Inst. Math. Acad. Sinica, v. 7, n. 3, p. 301-311, 1979.

CHEN, B. Y. On the total curvature of immersed manifolds, V: C-surfaces in Euclidean m-space. Bull. Inst. Math. Acad. Sinica, v. 9, n. 4, p. 509-516, 1981. 
CHERN, S. S.; LASHOF, R. K. On the total curvature of immersed manifolds. II, Michigan Math. J., v. 5, n. 1, p. 5-12, 1958.

HELFRICH, W. Elastic properties of lipid bilayers: theory and possible experiments, Z. Naturforsch, v. 28, p. 693-703, 1973.

HOU, Z. H. The total mean curvature of submanifolds in a Euclidean space, Michigan Math. J., v. 45, n. 3, p. 497-505, 1998.

OTSUKI, T. On the total curvature of surfaces in Euclidean spaces, Japan. J. Math, v. 35, p. 61-71, 1966.

WILLMORE, T. J. Mean curvature of immersed manifolds, An. Sti. Univ. "Al. I. Cuza" Iasi. Sect. I. a Mat, v. 14, p. 99-103, 1968. 